\title{
QCD '98: status of the power corrections
}

\author{
V.I. Zakharov ${ }^{\mathrm{a}}$ \\ ${ }^{a}$ Max-Planck Institut für Physik, 80805 Munich, Germany.
}

We review status of the power corrections in QCD. The topics include shape variables, unconventional $1 / Q^{2}$ corrections, tachyonic gluon mass as a fit parameter to the $1 / Q^{2}$ corrections. The selection of the material is determined mostly by results presented at the Conference QCD'98 (Montpellier, July 1998). Some background comments are also included.

\section{Introduction}

By power corrections to the parton model one understands terms of order $\left(\Lambda_{Q C D} / Q\right)^{n}$ where the characteristic mass scale $Q$ is assumed to be much larger than $\Lambda_{Q C D}$. At first sight, the inclusion of the power correction in any consistent analysis is very problematic since they are exponentially small in inverse running coupling, $\sim \exp \left(-n / 2 b_{0} \alpha_{s}\left(Q^{2}\right)\right)$, and, generally speaking, are subordinate to many orders in perturbative expansion which makes them extremely sensitive to an exact definition of $\alpha_{s}$ and so on.

Thus, to develop a phenomenology one assumes in fact that the power corrections are enhanced numerically and are responsible primarily for breaking of asymptotic freedom at intermediate $Q^{2}$. The first signal for that came from the QCD sum rules 11. The central object here is the correlators of the currents $j$ with various quantum numbers:

$\Pi_{j}\left(Q^{2}\right)=i \int \exp (i q x)\langle 0|T\{j(x), j(0)\}| 0\rangle$,

$\left(q^{2} \equiv-Q^{2}\right)$ and it is indeed known that the power corrections to the parton model predictions for $\Pi_{j}\left(Q^{2}\right)$ can be extracted with reasonable accuracy.

More relevant to the present discussion, the power corrections seem to be large in jet physics as well. In particular for a jet mass one has [2]

$$
\left(\frac{M_{\text {jet }}^{2}}{Q^{2}}\right)_{\text {non-pert }} \approx \frac{2\left\langle k_{\perp}\right\rangle}{Q} .
$$

where $Q$ is the jet energy and the intrinsic per- pendicular momentum of the partons is of order $\left\langle k_{\perp}\right\rangle \sim 0.5 \mathrm{GeV}$ so that the nonperturbative correction is quite large.

Most recently, the power corrections were discussed within the renormalon framework (for reviews and further references see [3]). Renormalons provide with a derivation of equations like (2) in a language directly related to the fundamental QCD.

Experimental and lattice data have been accumulated during last year and there have already been three talks at this Conference devoted entirely to the power corrections [- 6 . I feel that the relevant theoretical framework was basicly reviewed during the previous conferences in the same series [3]. Thus we add only a few remarks, mostly on new theoretical works which appeared meantime. Also, we include an overview of the original work [7].

\section{Shape variables.}

Data on $1 / Q$ corrections to the thrust, $\mathrm{C}$ parameter and heavy jet mass $M_{h}^{2}$ were obtained with high statistical accuracy and presented at this Conference [4]. The interest in these corrections, at least partly, was motivated by applications of the renormalon techniques [8 10]. In particular, the value of $n$ in $\left(\Lambda_{Q C D} / Q\right)^{n}$ is fixed reliably by the renormalons for each variable and the presence of the $\Lambda_{Q C D} / Q$ terms was demonstrated in case of the variables mentioned above. However, to find relations between various corrections one needs still models because in principle any number of the renormalon chains gives power 
corrections of the same order [11,3] and there is no way to evaluate all of them.

Proceeding to the models, within the universality picture [9.10] one keeps terms which contribute perturbatively in differential distributions of the variables as $\alpha_{s}^{m} l n^{k} Q$ and continues such terms to the infrared region (where, in general, they do not dominate any longer). As a result, all the $1 / Q$ corrections get expressed in terms of a universal factor $E_{\text {soft }}=\int d k_{\perp} \gamma_{e i k}\left(\alpha_{s}\left(k_{\text {perp }}^{2}\right)\right)$ where $\gamma_{e i k}$ is the so called eikonal anomalous dimension and the integral over the Landau pole is understood, say, as the principal value. Within this model one gets, in particular, [9]

$\langle 1-T\rangle_{1 / Q}=\frac{2}{3 \pi}\langle C\rangle_{1 / Q}$.

The data look as 但

$$
\begin{gathered}
\frac{1}{2}\langle 1-T\rangle_{1 / Q}=\frac{\text { const }}{Q}(0.511 \pm 0.009) \\
\frac{1}{3 \pi}\langle C\rangle_{1 / Q}=\frac{\text { const }}{Q}(0.482 \pm 0.008),
\end{gathered}
$$

and are in excellent agreement with the theory.

There are further theoretical predictions. In particular [9]:

$$
\begin{gathered}
\langle 1-T\rangle_{1 / Q}=\left(\frac{\left\langle M_{h}^{2}+M_{l}^{2}\right\rangle}{Q^{2}}\right)_{1 / Q} \\
\left(\frac{\left\langle M_{h}^{2}\right\rangle}{Q^{2}}\right)_{1 / Q} \approx\left(\frac{\left\langle M_{l}^{2}\right\rangle}{Q^{2}}\right)_{1 / Q}
\end{gathered}
$$

Although the equation (7) echoes the tube model (2) its derivation within another renormalonrelated approach [8] turned to be rather painful. The point is that this is an approach based on a single renormalon chain (or one-loop integration over the running coupling). Such an approximation is tempting because of its simplicity and various assumptions (like large $N_{f}$, naive nonabelianization, freezing of the coupling...) were tried to justify it. However, it is a basic fact about power corrections that the higher loops of the perturbative expansions, when projected onto the power corrections, all of them give contributions of the same order [11,3]. It was dramatically confirmed in case of the shape variables because in the one-loop approximation [8] one of the jets is deprived of any non-perturbative contributions and has mass strictly zero which cannot be true experimentally.

However, if one compares with experimental data, say, Eq. (3) then the both sides lose (for a two-jet event) a factor of 2 and the relation stays intact [9]. Only once one proceeds, e.g., to relations (7) the shortcomings of the model [8] become self-evident. During the last year the so called Milan factor [12] was discovered within the approach of Refs. W8. Which is, in essence, the observation that two loops contribute to the shape variables no less than one loop. The explicit form of the Milan factor emerges from a careful analysis of the infrared radiation on the two-loop level. As a result, in cases when same variables were considered within both approaches [9] and [8]

$(D W) \times($ Milan factor $)=($ universality $)$

where $(D W)$ stands for the original predictions of the model [8]. It might worth noting that the Milan factor does not necessarily keeps exactly the same contributions which are absorbed into the factor $E_{\text {soft }}$ (see above). However, the both models share the property that for a two-jet event nonperturbative contributions are the same for each of the jets. And the both derivations are still models, not a proof.

Experimentally,

$2\left(\frac{\left\langle M_{h}^{2}\right\rangle}{Q^{2}}\right)_{1 / Q}=\frac{\text { const }}{Q}(0.616 \pm 0.018)$

where the constant is the same as in Eqs. $(4,5)$. $\mathrm{kl}$ As is emphasized in [4] the experimental data on the jet broadening parameters, $B_{T}, B_{W}$ contradict the Milan factor (these variables have not been treated within the universality approach so far). On the other hand, there is no contradiction with hadronization models. My superficial judgment would be that the Milan factor will yield to the hadronization models as a result of further analysis. The reason is that the renormalons do incorporate the tube model as a hadronization model [9]. 


\section{Power corrections in DIS.}

Theory of the power corrections in deep inelastic scattering has been developed so far only on the one-loop level (i.e., a single renormalon chain) 13. Which might be not such a bad approximation (unlike the case of the $e^{+} e^{-}$annihilation, see above) since there is a single quark which radiates. And indeed first calculations [14] with inclusion of the Milan factor confirm these expectations. However, even on the one-loop level the Landau-pole parametrization of the power corrections produces in case of the $F_{L}$ structure function a result which is in variance with a single renormalon chain predictions 15$]$. Thus, the theoretical predictions appear to depend on details of the infrared cut off, the possibility always looming over the renormalon-based approaches. Also, the extraction of the numerical value for the power corrections in DIS at least in some cases depends crucially on the subtraction of the perturvative pieces 16.

A new and systematic extraction of the power correction to the non-singlet structure function $F_{2}$ from the experimental data was presented at this Conference [5]. The power corrections were studied as a function of $n$ where $n$ is the number of the moment from the structure function $F_{2}(x)$ and the $n$-dependence appears to agree with the renormalon-based predictions.

\section{Power corrections beyond the OPE.}

So far we discussed power corrections associated with infrared region. Although the theoretical predictions may vary in this case as well, the physical picture behind the IR corrections is simply the growth of the running coupling at large distances and this mechanism seems safe.

During the last couple of years unconventional $1 / Q^{2}$ corrections which go beyond this simple picture have also been discussed. At this Conference results on measuring $1 / Q^{2}$ terms in $\alpha_{s}$ have been presented [6]. Papers in Ref. [6] contain not only results of the lattice measurements but an exposure of the theoretical framework as well and we would not like to overlap with this discussion. The $1 / Q^{2}$ corrections are viewed in Ref. [6] as arising within the dispersive approach, or from removal of the Landau from the running coupling 17.18]. In fact, there is another way to introduce the $1 / Q^{2}$ corrections which go beyond the standard OPE, that is via the UV renormalons [19]. It has not been clarified yet, whether there is any connection between the two ways of viewing the unconventional $1 / Q^{2}$ corrections.

The leading ultraviolet renormalon brings perturbative expansions of the form:

$$
\left(\sum_{n} a_{n} \alpha_{s}^{n}\left(Q^{2}\right)\right)_{U V}=\sum_{n} n !\left(-b_{0}\right)^{n} \alpha_{s}^{n}\left(Q^{2}\right) \text {. }
$$

Because of the $n$ ! divergence of the expansion coefficients the sum (10) is not defined. It is quite common to assume however that the Borel summation is the correct recipe to deal with the divergence. This hypothesis amounts to replacing the growing branch of the perturbative expansion (10) by its integral representation:

$\sum_{N_{c r}}^{\infty} n !\left(-b_{0}\right)^{n} \alpha_{s}^{n} \rightarrow \int \frac{\left(\alpha_{s} b_{0} t\right)^{N_{c r}} \exp (-t) d t}{1+\alpha_{s} b_{0} t}$

where $N_{c r}=1 / b_{0} \alpha_{s}$ is the value of $n$ for which the absolute value of the terms in the series reaches its minimum and the right-hand side is readily seen to be of order $\frac{\Lambda_{Q C D}^{2}}{Q^{2}}$ :

$\frac{1}{2}\left(a_{n} \alpha_{s}^{n}\left(Q^{2}\right)\right)_{n=N_{c r}} \sim \frac{\Lambda_{Q C D}^{2}}{Q^{2}}$.

It is amusing to observe that this correction comes from huge virtual momenta of order $p^{2} \sim Q^{2}$. $\exp \left(N_{c r}\right)$.

Thus, if the theory is defined with an intrinsic UV cut off, like the lattice theory, then the contribution of the UV renormalon may well be irrelevant. Then an alternative language of dispersive approach to the running coupling [17,18] can be utilized. Within this approach, one may think in terms of the removal of the Landau pole from the coupling:

$\frac{1}{\ln Q^{2} / \Lambda_{Q C D}^{2}} \rightarrow \frac{1}{\ln Q^{2} / \Lambda_{Q C D}^{2}}-\frac{\Lambda_{Q C D}^{2}}{\left(Q^{2}-\Lambda_{Q C D}^{2}\right)}$

To justify this modification of the coupling one argues that to any finite order in perturbation 
theory the coupling satisfies dispersion relations with cuts at physical $s>0$. The procedure, which has been discussed since the fifties, clearly introduces a $\Lambda_{Q C D}^{2} / Q^{2}$ correction to $\alpha_{s}$ at large $Q^{2}$.

The bulk of the discussion is build on the belief that the replacement (13) results in a new universally defined coupling which can be used then for phenomenological purposes. However, it was argued in Ref. [18], and I still consider the argument convincing, that the dispersive approach does not end up with a universal redefinition of the coupling. Instead, all the terms in $\alpha_{s}\left(Q^{2}\right)$ perturbative expansion collapse to the same order power correction once the Landau pole is removed from the dispersive representation for $\alpha_{s}^{n}\left(Q^{2}\right)$. In more detail [20]:

$\sum_{n} a_{n} \alpha_{s}^{n}\left(Q^{2}\right) \rightarrow \sum_{n} a_{n} \alpha_{s}^{n}\left(Q^{2}\right)-\sum_{n} \frac{a_{n}}{n ! b_{0}^{n}} \frac{\Lambda_{Q C D}^{2}}{Q^{2}}$.

Thus to establish a connection (if any) of the removal of the pole from dispersion representations with the UV renormalons one has to consider the whole series in $\alpha_{s}\left(Q^{2}\right)$. In particular, let us apply (14) to the case $a_{n}=n !\left(-b_{0}\right)^{n}$. Then the resulting power correction, $\sum_{n}(-1)^{n} \Lambda_{Q C D}^{2} / Q^{2}$, is still poorly defined. If, however, we invoke the Borel summation,

$$
\left(\sum_{n}(-1)^{n} \frac{\Lambda_{Q C D}^{2}}{Q^{2}}\right)_{\text {Borel }}=\frac{1}{2} \frac{\Lambda_{Q C D}^{2}}{Q^{2}},
$$

then the power corrections resulting from the procedures (14) and (11) are the same. This example demonstrates that there could be a close connection between the Borel summation of the UV-renormalon series (11) and the removal of the Landau pole (13) from dispersion relations. Moreover, one may argue that if introduction of the UV cut off into the theory removes the UV renormalon, the coupling should be redefined along the lines of Eq. (13) so that the theory with and without the UV cut off remains the same at relatively low momenta on the level of the $1 / Q^{2}$ terms. Thus, one is invited to generalize the logic of the renormalization group to include the $1 / Q^{2}$ corrections as well.

Let us now turn to another issue, namely the physical meaning of the replacement (13). To this end we need a more precise definition of $\alpha_{s}\left(Q^{2}\right)$ and we assume now that $\alpha_{s}\left(Q^{2}\right)$ is defined in terms of the Fourier transform of the potential of heavy quarks $V(r)$ 21]. It is obvious then that the change (13) results in a linear correction to the potential at short distances $r \ll \Lambda_{Q C D}^{-1}$ :

$\lim _{r \rightarrow 0} V(r)=-\frac{4 \alpha_{s}(r)}{3 r}+($ const $) r$.

On the other hand, within the so to say standard QCD the leading power correction at short distances is of order $r^{2}$. This conclusion is based solely on the assumption that the nonperturbative fluctuations in QCD are of large scale, $\sim \Lambda_{Q C D}^{-1}$ (for references and further explanations see [21]). Thus, introduction of the new corrections (13) assumes small-size nonperturbative fields and a particular picture of the vacuum properties which results in this effect is described in Ref [21]. Arguments that at least for some definitions of $\alpha_{s}$ the $1 / Q^{2}$ piece can be associated only with short distances were also given most recently in Ref. [22].

\section{Tachyonic gluon mass.}

The $1 / Q^{2}$ corrections discussed in the previous section go beyond the standard OPE. Detection of the new type corrections through phenomenology would be of great interest. In this section we will discuss phenomenology in terms of a tachyonic gluon mass which is assumed to mimic the short-distance nonperturbative effects [7].

First, let us note that not all the $1 / \vec{Q}^{2}$ corrections in QCD are associated with short distances. For example, in case of DIS the $1 / Q^{2}$ corrections are coming from the IR region and perfectly consistent with the OPE. Thus, the class of theoretical objects for which an observation of the $1 / Q^{2}$ corrections would signify going beyond the OPE is limited. One example was the potential $V(r)$ discussed above. Other examples are the correlator functions (11) where the IR-sensitive power corrections start with $Q^{-4}$ terms [1].

Thus, we concentrate on this set of variables and an interesting question is whether there is room for introduction of sizable non-standard $1 / Q^{2}$ corrections. The answer seems to be in pos- 
itive [7]. First of all, the potential (16) itself has been measured on the lattice down to fairly short distances [23 and there is no sign of the change from the linear term $k r$ (where $k$ is the string tension) to a quadratic correction $c r^{2}$ as is predicted by the OPE. Thus at this time one is free to speculate that the form (16) continues to $r \rightarrow 0$ with const $\sim k \approx 0.2 \mathrm{GeV}^{2}$.

Even if one accepts this assumption, it is far from being trivial to relate the constant $k$ to the scale of the $1 / Q^{2}$ corrections to other quantities. Qualitatively, however, one may hope that introduction of a tachyonic gluon mass at short distances would imitate the effect of the $\Lambda_{Q C D}^{2} / Q^{2}$ corrections. Indeed, the linear term in (16) and with const $\sim k$ can be imitated [20] by the Yukawa potential with a gluon mass $\lambda$ :

$\frac{4 \alpha_{s}}{6} \lambda^{2} \sim-k$.

This picture with a tachyonic gluon mass can be consistently used at one-loop level as well.

Of course Eq. (17) may serve only for a rough estimate. First of all, there are no error bars on the value of $k$ at short distances since the measurements [23] were not dedicated to the short distances. Moreover, Eq. (17) assumes that the short-distance potential is due to a vector-like exchange while at large distances the $k r$ term corresponds to a scalar exchange and there is no evidence for a change [23]. However, the reservations (which are numerous) should not mask the fact that the gluon mass is large according to (17). And the real question is [7] whether a kind of large tachyonic gluon mass is admitable in view of the known properties of $\Pi_{j}\left(Q^{2}\right)$.

One of the basic quantities to be determined from the theory is the scale at which the parton model for the correlators (11) gets violated considerably via the power corrections. Technically, one studies usually $\Pi\left(M^{2}\right)$ where [1]

$\Pi_{j}\left(M^{2}\right) \equiv \frac{Q^{2 n}}{(n-1) !}\left(\frac{-d}{d Q^{2}}\right)^{n} \Pi_{j}\left(Q^{2}\right)$

in the limit where both $Q^{2}$ and $n$ tend to infinity so that their ratio $M^{2} \equiv Q^{2} / n$ remains finite. Moreover, within the standard OPE the correla- tors $\Pi\left(M^{2}\right)$ at large $M^{2}$ is represented as:

$$
\begin{array}{r}
\Pi_{j}\left(M^{2}\right) \approx(\text { parton model }) . \\
\left(1+\frac{a_{j}}{\ln M^{2} / \Lambda_{Q C D}^{2}}+\frac{b_{j}}{M^{4}}+O\left(\left(\ln M^{2}\right)^{-2} M^{-6}\right)\right)
\end{array}
$$

where the constants $a_{j}, b_{j}$ depend on the channel, i.e. on the quantum numbers of the current $j$. The terms of order $1 / \ln M^{2}$ and $M^{-4}$ are associated with the first perturbative correction and the gluon condensate, respectively. To characterize the scale of the power corrections, one may introduce [24] the notion of $M_{c r i t}^{2}$ which is defined as the value of $M^{2}$ at which the power corrections become, say, $10 \%$ from the unit. The meaning of $M_{c r i t}^{2}$ is that at lower $M^{2}$ the power corrections blow up.

In the $\rho$-channel,

$M_{\text {crit }}^{2}(\rho-$ channel $) \sim 0.6 \mathrm{GeV}^{2}$

which is determined by the value of the gluon condensate $<\alpha_{s}\left(G_{\mu \nu}^{a}\right)^{2}>$ and agrees well with independent evaluation of $M_{\text {crit }}^{2}$ from the experimental data on the $e^{+} e^{-}$annihilation [1] . In the language relevant to the present review, the gluon condensate represents IR renormalons.

If one proceeds to other channels, in particular to the $\pi$-channel and to the $0^{ \pm}$-gluonium channels, nothing special happens to $M_{c r i t}^{2}$ associated with the IR renormalons. However, it was determined from independent arguments that the actual values of $M_{c r i t}^{2}$ do vary considerably in these channels [24]:

$$
\begin{aligned}
M_{\text {crit }}^{2}(\pi-\text { channel }) & \geq 1.8 \mathrm{GeV}^{2} \\
M_{\text {crit }}^{2}\left(0^{ \pm}-\text {gluonium channel }\right) & \geq 15 \mathrm{GeV}^{2} .
\end{aligned}
$$

These lower bounds on $M_{\text {crit }}^{2}$ are obtained from the values of $f_{\pi}$ and of the quark masses in the pion channel, and from a low-energy theorem in the gluonic channel. Such values of $M_{c r i t}^{2}$ cannot be recoinciled with the IR renormalons.

Now, a new term proportional to $\lambda^{2}$ is added to the theoretical side of $\Pi_{j}\left(M^{2}\right)$ which becomes:

$\Pi\left(M^{2}\right) \approx($ parton model $)\left(1+\frac{a_{j}}{\ln M^{2}}+\frac{b_{j}}{M^{4}}+\frac{c_{j}}{M^{2}}\right)($

where $c_{j}$ is calculable in terms of $\lambda^{2}$ [7]:

$c_{\pi} \approx 4 c_{\rho}=\frac{4 \alpha_{s}}{3 \pi} c_{\text {gluonium }}=\frac{4 \alpha_{s}}{\pi} \lambda^{2}$. 
Phenomenologically, in the $\rho$-channel there are severe restrictions [25] on the new term $c_{j} / M^{2}$ :

$c_{\rho} \approx-(0.03-.07) \mathrm{GeV}^{2}$.

Remarkably enough, the sign of $c_{\rho}$ does correspond to a tachyonic gluon mass (if we interpret $c_{\rho}$ this way). Moreover, when interpreted in terms of $\lambda^{2}$ the constraint (26) does allow for a large $\lambda^{2}$, say, $\lambda^{2}=-0.5 G e V^{2}$.

As for for the $\pi$-channel one finds now a new value of $M_{\text {crit }}^{2}$ associated with $\lambda^{2} \neq 0$ :

$M_{\text {crit }}^{2}(\pi-$ channel $) \approx 4 \cdot M_{\text {crit }}^{2}(\rho-$ channel $)(27)$

which fits nicely the Eqs. (21) and (22) above. Moreover, the sign of the correction in the $\pi$ channel is what is needed for phenomenology 24. Fixing the value of $c_{\pi}$ to bring the theoretical $\Pi_{\pi}\left(M^{2}\right)$ into agreement with the phenomenological input one gets

$\lambda^{2} \approx-0.5 \mathrm{GeV}^{2}$.

Finally, we can determine the new value of $M_{c r i t}^{2}$ in the scalar-gluonium channel and it turns to be what is needed for the phenomenology, see Eq (23). Thus, qualitatively the phenomenology with a tachyonic gluon mass which is quite large numerically stands well to a few highly nontrivial tests.

It is worth emphasizing that the $\lambda^{2}$ terms represent nonperturbative physics and limit in this sense the range of applicability of pure perturbative calculations. This nonperturbative piece may well be much larger than some of the perturbative corrections which are calculable and calculated nowadays.

Further crucial tests of the model with the tachyonic gluon mass could be furnished with measuremants of various correlators $\Pi_{j}\left(M^{2}\right)$ on the lattice.

\section{Conclusions}

We discussed briefly the status of the power corrections both from IR and UV regions. The infrared renormalons produce a picture close to the hadronization models. Phenomenologically, there is room for a new $\Lambda_{Q C D}^{2} / Q^{2}$ correction coming from the ultraviolet. If confirmed, such a correction would be of great interest.

I would like to acknowledge gratefully the hospitality of the Centre National de la Recherche Scientifique (CNRS), and especially of S. Narison, during the stay at the University of Montpellier where part of this work was done. I am also grateful to R. Akhoury, G. Bali, K.G. Chetyrkin, and S. Narison for interesting discussions and remarks.

\section{REFERENCES}

1. M.A.Shifman, A.I. Vainshtein, V.I. Zakharov, Nucl. Phys. B147 (1979) 385, 448.

2. B. R. Webber, hep-ph/9411384.

3. R. Akhoury, V.I. Zakharov, hep-ph/9610492, hep-ph/9710257.

4. P.A.M. Fernăndez, hep-ex/9808005; P.A.M. Fernăndez et. al., hep-ex/9807007.

5. S. Liuti, hep-ph/9809248.

6. G. Burgio et. al., hep-ph/9808258, hep$\mathrm{ph} / 9809450$.

7. K.G. Chetyrkin, S. Narison, V.I. Zakharov, hep-ph/9811275.

8. B.R. Webber, Phys. Lett. B339 (1994) 148; Yu. L. Dolshitzer, B.R. Webber, Phys. Lett. B352 (1995) 451; Yu. L. Dokshitzer, G. Marchesini, B.R. Webber, Nucl. Phys. B444 (1995) 602.

9. R. Akhoury, V.I. Zakharov, Phys. Lett. B357 (1995) 646; Nucl. Phys. B465 (1996) 295.

10. G.P. Korchemsky, G. Sterman, hep-ph/9505391.

11. A.I. Vainshtein, V.I. Zakharov, Phys.Rev.Lett. 73 (1994) 1207.

12. A. Lucenti et. al.Nucl.Phys. B511 (1998)396. 13. E. Stein et.al., Phys. Lett. B376 (1996) 177, hep-ph/9803342.

14. M. Dasgupta, B.R. Webber, hep-ph/9809247.

15. R. Akhoury, V.I. Zakharov, hep-ph/9701378.

16. A. Kataev et. al., hep-ph/9706534.

17. G. Grunberg, hep-ph/9705290, hep-ph/ 9711481.

18. R. Akhoury, V.I. Zakharov, hep-ph/9705318.

19. V.I. Zakharov Nucl. Phys. B385 (1992) 452; S. Narison, Phys.Lett. B300 (1993) 293; A.I. Vainshtein, V.I. Zakharov, Phys.Rev. 
D54 (1996) 4039; K. Yamawaki, V.I. Zakharov, hep-ph/9406399, hep-ph/9406373; G. Altarelli, P. Nason, G. Ridolfi, Z. Phys. C68 (1995) 257; S. Peris, E. de Rafael, Nucl. Phys. B500 (1997) 325; M. Beneke, V.M. Braun, N. Kivel, Phys. Lett. B404 (1997) 315.

20. V.I. Zakharov, hep-ph/9802416

21. R. Akhoury, V.I. Zakharov hep-ph/9710487.

22. M. Beneke, V.M. Braun, N. Kivel, hepph/9809287.

23. G.Bali et. al. hep-lat/950617; Phys.Rev. D55 (1997) 5309.

24. V.A. Novikov et.al., Nucl. Phys. B191 (1981) 301

25. S. Narison, Phys.Lett. B300 (1993) 293. 